Accepted Manuscript (AM) version of article

It is authorized for deposition in a non-commercial subject repository on acceptance of publication by the terms of the copyright.

Formatted by the author to enhance readability. 


\title{
Beyond Writing: The Development of Literacy in the Ancient Near East
}

\author{
Karenleigh A. Overmann
}

\begin{abstract}
Previous discussions of the origins of writing in the Ancient Near East have not incorporated the neuroscience of literacy, which suggests that when southern Mesopotamians wrote marks on clay in the late-fourth millennium, they inadvertently reorganized their neural activity, a factor in manipulating the writing system to reflect language, yielding literacy through a combination of neurofunctional change and increased script fidelity to language. Such a development appears to take place only with a sufficient demand for writing and reading, such as that posed by a state-level bureaucracy; the use of a material with suitable characteristics; and the production of marks that are conventionalized, handwritten, simple, and non-numerical. From the perspective of Material Engagement Theory, writing and reading represent the interactivity of bodies, materiality, and brains: movements of hands, arms, and eyes; clay and the implements used to mark it and form characters; and vision, motor planning, object recognition, and language. Literacy is a cognitive change that emerges from and depends upon the nexus of interactivity of the components.
\end{abstract}

Neuroscience and cognitive psychology provide insight into how literacy changes the brain, used here to interpret the archaeological and textual records of the Ancient Near East (ANE) and examine the development of literacy. Writing is differentiated from literacy, and the neurofunctional reorganization involved in literacy and the effects of handwriting on cognitive functioning are briefly described. Also depicted are the ways in which writing systems change into scripts and the contribution of materiality to change in both brains and script. Cumulatively, these factors suggest how literacy develops in original contexts, including the specific circumstances that produce it, how it might be discerned through script and other change, and why numerical notations alone cannot cause it.

The framework used herein is Material Engagement Theory (MET; Malafouris 2013), which views cognition as a system comprised of brains, bodies, and materiality in dynamic interaction with one another, interactivity with the potential to change both systemic outcomes and individual components. Through the MET lens, writing and reading, whether literate or not, represent the interactivity of psychological processes like vision and motor planning; behaviours involving movements of the hands, arms, and eyes; and materials like clay and characters of writing and script. Should this interactivity occur with sufficient repetition over time, brains reorganize to become literate, and writing acquires the capability to express language. Given an expressive script (i.e., one capable of representing the lexicon, grammar, and syntax of a language with sufficient semantic and phonetic clues to link written expressions to a specific language), the brain reorganization that is literacy is then instilled by teaching individuals to handwrite characters and recognize them in combinations of increasing complexity. However, when a writing system is inexpressive, it may require centuries to acquire the capability, and it does so in interaction with brains that are initially not literate. Literacy, then, is a cognitive change that emerges from, and which depends upon, certain behavioural-psychological-material 
interactions over time. There are two significant differences between us and the Mesopotamians ${ }^{1}$ of the late-fourth millennium in this regard: First, we know what literacy is when we engage in the behaviours producing it, while they did not. Second, our scripts possess the requisite fidelity to language, while archaic Mesopotamian writing did not.

\section{Writing and literacy}

To understand the development of literacy, it is important to differentiate it from functional reading and writing; this will be illustrated with the foreigner's experience of Nihongo (the Japanese language) to provide insight into reading an unknown script, essentially a mnemonic exercise similar to that conjectured for early writing (Diakonoff 1976). English-speaking visitors to Japan quickly encounter a complex mixture of scripts: Kanji, ornate logograms originally developed to write Chinese; hiragana, indigenous signs for syllables used to specify Nihongo readings of Kanji; and katakana, the identical syllables in different signs used only to write foreign words. Though these various signs function effectively as a script within an existing system of literacy for the Japanese, they do not have the same effect for foreigners, whose situation is somewhat similar in this regard to that of fourth-millennium Mesopotamians confronted with archaic writing (although foreigners' literacy in English and understanding of what literacy is clearly differentiate the two).

Foreigners might learn to recognize Kanji through associations of signs with their meanings in English, a language that Kanji does not express. Given the visual complexity of some of the signs, foreigners might also compare them to an explained list in a guidebook, something that also depends upon their literacy in English. Similarly, foreigners who learn the sign-sound associations of katakana might also become able to read a few words on menus and public signs. Unlike Kanji recognition, a matter of memorizing sign-meaning associations, understanding words in katakana requires remembering sign-sound associations and sounding out the syllables (Fig. 1). While signs whose semantic or phonetic associations have not been learned remain meaningless to foreigners, the abilities to recognize Kanji and sound out katakana constitute a functional ability to read, albeit one quite different from that involved in reading the other words on this page (hereafter, the first will be differentiated as functional reading and writing, the second as literacy or true literacy). The contrast between them underlies the present review of how literacy may have developed from functional reading and writing in the ANE, something that required change in both brains and writing.

\begin{tabular}{|cccc|}
\hline & Kanji & & Katakana \\
出口 & 横須賀 & ハンバーガー & サービス \\
& & ha-n-baa-gaa & saa-bi-st \\
'exit' & 'city of Yokosuka' & 'hamburger' & 'service' \\
\hline
\end{tabular}

Figure 1. For someone not literate in Nihongo, reading Kanji requires learning sign-meaning associations or comparing signs to known exemplars, similar to the mnemonic function of early ANE picto-/ideographs. Reading katakana requires learning sign-sound associations and application rules; where katakana form a systematic syllabary and are used to spell entire words, the earliest ANE syllabic signs were used to specify aspects of logograms (e.g. verbal form) and did not represent Sumerian phonemes with precision (Diakonoff 1976). 
Certainly, there are more kinds of literacy than these. A third is one wherein an individual lacks proficiency, from either insufficient training or inadequate practice; examples include children learning to read in modern classrooms and monks working as copyists in medieval scriptoriums. ${ }^{2}$ These too represent functional reading and writing, though there is a critical difference: Both student and copyist work with scripts, writing systems that have become highly expressive of language (hereafter, these will be differentiated as writing, which lacks language expressiveness, and script, which has acquired it). Scripts did not—indeed, could not—exist when writing was first invented; the expressive power of archaic proto-cuneiform was so minimal that the language it represented cannot be identified with certainty, though its inventors undoubtedly spoke one (Hyman 2006), possibly Sumerian (Veldhuis 2014; but also see Englund 1998).

To enable literacy, a writing system must be capable of representing the lexicon, grammar and syntax, and parts of speech with both semantic and phonetic clues to a specific language (Vanstiphout 1995). When it does not, the situation differs from that of the foreigner who recognizes Kanji and katakana through meaning and sound associations, since the foreigner's guidebook explains Nihongo signs in English accessible through literacy. In comparison, lexical lists in archaic writing, though often described as 'dictionaries' and 'teaching tools' (Veldhuis 2014, 16, 56), were probably more like codebooks, training more about memorizing sequences of signs (Wagensonner 2010). Consulting a lexical list to resolve an unknown sign perhaps involved finding it on a list and accessing its meaning through a memorized sequence, usage that does not resemble consulting a dictionary in the way we understand it today.

A classic definition of literacy is the idea that it has gradations. Three such gradations are functional literacy, knowing enough signs 'to write or read a letter or an ordinary business document'; technical literacy, involving arcane signs or jargon 'more difficult to read for the untrained, but not for the specialist'; and scholarly literacy, knowing 'the writing system for its own sake, collecting all and impossible readings of each sign and sign combination, and studying the history of its use and paleography' (Veldhuis 2011, 71-4; notably, Veldhuis' literate gradations refer to the Old Babylonian period, perhaps the first phase of writing that was truly literate). These are primarily differentiated by the number of signs (and related concepts) that someone needed to know to perform at a particular level. Gradated literacy, especially its conceptualization of functional literacy, is orthogonal to the distinction proposed here, which focuses on essential changes in brain and the writing system that the notion of gradation fails to capture.

MET allows us to conceptualize literacy as a cognitive system, consider how the system changes over time both in its overall functionality and individual components, and compare system characteristics at one time to those of another. Two such changes over time, already mentioned, are brain functionality (literate or not) and writing expressiveness (expressive or not). ${ }^{3}$ Another is the degree of accessibility between archaic, functional reading and writing and the later, truly literate system. Going back to the Nihongo example, acquiring the foreigner's knowledge of Kanji and katakana is a relatively straightforward matter of memorizing signmeaning and sign-sound associations, as well as rules for their application. Archaic writing would have been acquired with similar ease, a matter of access to the community and materials of writing and sufficient training to decode the signs; it would not have involved significant neural reorganization and did not depend on writing's expressiveness.

Against the relative ease of acquiring the functional ability to read and write, true literacy involves not only knowing the language but teaching the brain to recognize its written form, 
something that also depends on the script having the requisite fidelity to language. However, unlike the scripts used to read and write Nihongo by literate Japanese, (proto-)cuneiform lacked the expressive power needed for literacy, a quality it would develop over centuries of interaction with neurofunctional changes in the brain.

\section{Brains in literacy: Neurofunctional reorganization}

Literacy involves multiple regions of the brain (Dehaene et al. 2010; Nakamura et al. 2012; Perfetti 2003; Perfetti \& Tan 2013), activity that appears fairly consistent across individuals, languages, cultures, and writing systems (Bolger et al. 2005; Carreiras et al. 2007; Frost 2012). One consistently activated brain region is the Visual Word Form Area (VWFA), a part of the fusiform gyrus in the temporal lobe that evolved for, and remains involved in, recognizing faces and objects (Price \& Devlin 2003; Vogel et al. 2014). Its activity in literacy represents significant neurofunctional change following from a specific engagement of materiality: reading and (hand)writing marks on some material form. Before even the concept of literacy existed, as was originally the case in the ANE, Mesopotamians made marks on clay to record and communicate information. Their fusiform gyri, as our do today, apparently had sufficient plasticity to respond, not just to faces and objects, but to cultural inputs in the form of written marks as well (Dehaene \& Cohen 2007; 2011; McCandliss et al. 2003). The VWFA identifies words from script features and demonstrates 'a clear superiority for words over pictures' (Dehaene \& Cohen 2011, 255), proficiency for recognizing signs that develops through training and practice. This neurofunctional change is identified as an example of neuronal recycling, in which a cultural invention co-opts an existing brain function (Cohen \& Dehaene 2004).

Evidence for neuronal recycling comes from the functionality shared in recognizing both faces/objects and written words from their local and global features (Adelman et al. 2010; Pelli et al. 2003; Fig. 2a). Feature recognition in cuneiform works similarly: words in cuneiform are recognized by their local and global features (individual wedges and their contexts; Fig. 2b). As an example of context, 'the sign NI, when preceded by BE, will be read /LI/; in Akkadian, bēli means "my lord." But in the sequence PA NI, NI will be read /NI/, panī meaning "my face", (Charpin 2010, 9). Feature recognition is likely the reason that scripts are able vary so widely in form (i.e. the different appearances of cuneiform, hieroglyphs, Chinese, and Mayan): the potential for a written object's being recognized through its features is relatively independent of its actual form. ${ }^{4}$ Since characters become recognized through combinations of local and global features, the need to preserve the original iconic appearances of pictographic images may be relaxed, a phenomenon inherent in the chronologies of individual (proto-)cuneiform signs (Fig. 3) that argues against the idea that writing systems and scripts 'do not develop' from pictographs (Daniels 1996, 3; emphasis added).

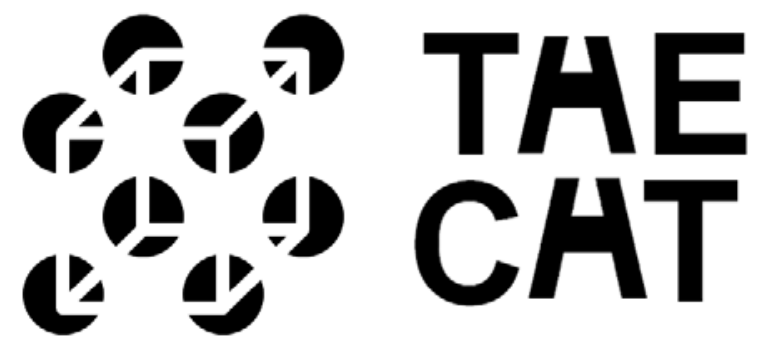

Figure 2a. Neuronal recycling is evident in the shared functionality of recognizing objects (left) and words (right) from a mix of local and global features. At left, readers should see a cube constructed from angular lines inside circles (local features) and their relations to one another (global features). At right, most will see the words 'the cat'; identically malformed middle characters are read as " $H$ " (top) and " $A$ " (bottom), interpretations derived from the characters themselves (local features) 
and their contexts within the words (global features).

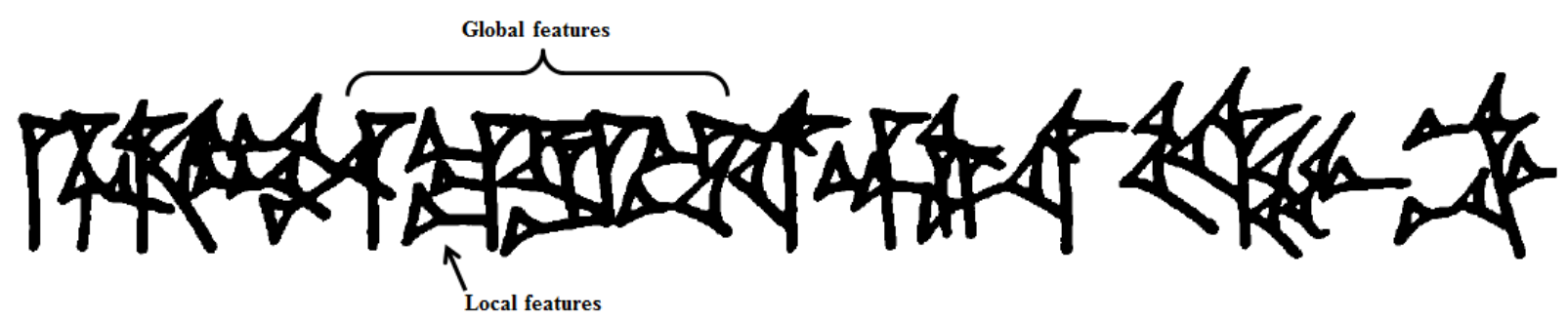

Figure 2b. Feature recognition for cuneiform works by associating meanings and sounds with wedges (local features) in combinations and contexts (global features). The text is from an Old Babylonian letter (obverse, line 10); adapted from Saggs (1960).

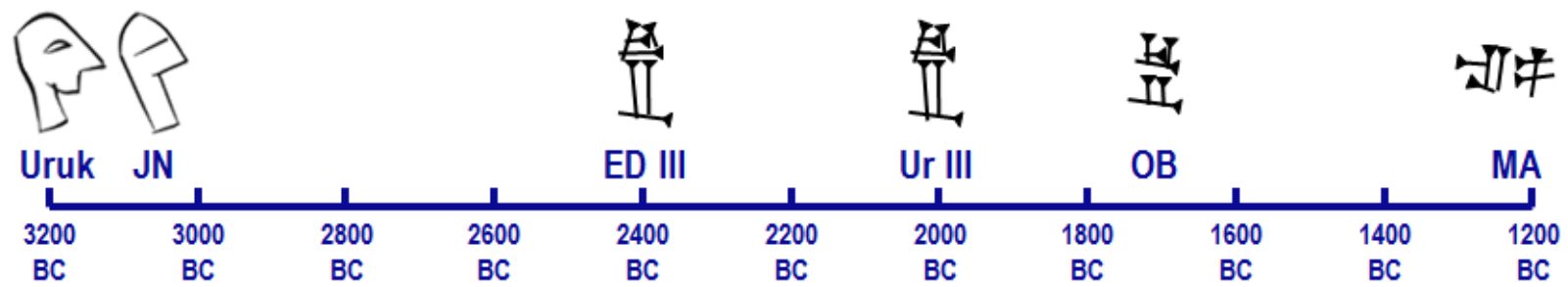

Figure 3. Chronology of the sign SAG. Source: redrawn from Nissen et al. (1993). JN = Jemdet Nasr; ED = Early Dynastic; $O B=$ Old Babylonian; $M A=$ Middle Assyrian.

Similarly, reading appears to have inherited constraints from face/object recognition, like the limit on the number of items that can be simultaneously attended. Known as subitization, the constraint may limit most characters to three strokes (Changizi \& Shimojo 2005). This constraint may be more apparent in writing systems like the Latin alphabet than others; Kanji signs, for example, often have many more than three strokes (Coltheart 2014). Where this is the case, distinct neural activation patterns suggest differences in phonological processing (Tan et al. 2005) and working memory (Cantlon \& Brannon 2007), functions reasonably related to the additional demands imposed by the greater visual complexity of the characters. The number of strokes in cuneiform characters suggests they would have been similar to Kanji in this regard.

The VFWA interacts with brain areas involved in the planning and execution of the motor movements producing handwriting (Exner's area) and speech (Broca's area), as well as comprehending speech (Wernicke's area and its epicenter, the planum temporale, which provides for the auditory representation of phonemes) (Pegado et al. 2014). The interaction with Wernicke's area is particularly important, as it informs a writing system's ability to represent language sounds, the phonetic function that Daniels (1996) identifies as a sine qua non characteristic of script. As reading and writing are practiced, brain form changes: white matter density increases, as does inter-hemispheric connectivity (Carreiras et al. 2009). Brain function changes too (e.g. VFWA training and inter-regional coordination). Feature recognition of written marks is, however, distinct from associating visual forms with the meanings and sounds of language, functions supported by the coordination between the VFWA and Broca's/Wernicke's areas. Similarly, the integration of Exner's area into the inter-regional coordination that 
characterizes literacy may provide 'a core recognition of the gesture in the written word' (Dehaene, quoted in Konnikova 2014).

In both original contexts and subsequent enculturation into existing systems of literacy, functional reading and writing precede literacy, suggesting the implicit behavioural-materialpsychological interactivity repurposes neural activity and trains the regions supporting motor movements, visual perception, object recognition, and language to coordinate with each other. Some inter-regional coordination surely also occurs during functional reading and writing (i.e. the word for 'sheep' would become associated with the ideograph for sheep). However, these effects alone would not produce literacy: unless and until writing is performed with sufficient repetition over time, literacy will not develop. This is seen when children acquire literacy: they must both learn and practice. Further, the writing system must develop the required fidelity to language; without expressive power, the writing system involves only simple associations: this sign has that meaning or sound.

When the fusiform gyrus (VWFA) is trained, it interacts with the parts of the brain that control handwriting and produce and understand speech. This interactivity differentiates a literate brain from one that is not literate. The question is, what are the specific circumstances needed to train these regions to recognize written objects by their features and interact so to associate the meanings and sounds of language with features of written objects? Obviously, the Mesopotamians had no prior concept of literacy when they first wrote marks on clay. But, when they repeated this behavior sufficiently_enough to meet the needs of administering large urban centers with extensive trade, managed agriculture, massive construction, and considerable military and slave labor forces - their brains began to reorganize and their writing system began to change, unintended and unforeseen consequences of their behaviour.

\section{Bodies in literacy: Training, practice, and handwriting effects}

No specific training is required for someone to acquire language. A child immersed from birth in a community of speakers begins speaking effortlessly at a very young age and becomes competent despite being exposed to only a limited sample of language (poverty of stimulus: see Chomsky 2012). By comparison, literacy must be learned: people do not acquire it simply from being around literate people. Moreover, the acquisition of literacy has specific effects on the form and function of brains and scripts, effects acquired through behavioural interactions with the community and materiality of writing and intensified through practice. Interestingly, over the centuries it took for cuneiform to develop language fidelity, the script also became increasingly opaque to non-initiates, even as literacy itself became a more widespread phenomenon. ${ }^{5}$ Mastering it was no longer a simple matter of decoding sign meanings and sound conventions but entailed reorganizing the brain through specific training and practice, without which the script was just so many undifferentiable marks on clay. The effort of training and practice, and the script's relative meaninglessness without it, would have effectively limited literacy to those with the necessary opportunities and skills and would have been a factor in the formalization of such training.

Understanding how literacy emerged from the activity of making marks on clay requires considering the effects of handwriting. As the hand is trained to make the fine movements that produce characters, vision is trained to work with the hand, improving hand-eye coordination in a way specific to literacy (as opposed to other skilled activities, e.g. flintknapping). Hand-eye coordination in literacy involves Exner's area, the brain region implicated in planning motor 
movements that are complex and finely coordinated; it is located in the premotor cortex and supplementary motor area of the frontal cortex, just above Broca's area. Exner's area is thought to support literacy by recognizing the gestural traces in writing; damage to the region is associated with alexia and agraphia, respectively, difficulty in reading and writing (Heimann et al. 2013). The loss of functionality that results when Exner's area is damaged is analogous to the loss of language functions (aphasia) from damage to Broca's and Wernicke's areas for producing and comprehending language.

There are other handwriting effects: as hand-eye coordination improves, so too does the ability to recognize characters across greater ambiguity of form; in addition, the ability to recall written material is improved, thought to occur through deeper levels of processing (James \& Engelhardt 2012; Longcamp et al. 2005; Mueller \& Oppenheimer 2014; Sülzenbrück et al. 2011). Handwriting effects have an important implication for the circumstances that produce literacy: they suggest that characters must be handwritten and involve the repetition of characters, not produced by techniques involving other fine motor movements (e.g. in lapidary work, the difference between chipping an edge to produce a bird figure and writing the character 'bird' repeatedly). Handwriting affords the repetition of characters at a volume that allows the effects to occur. Handwriting also facilitates the manipulation of character forms in ways that enable writing to become script ${ }^{6}$ (i.e. in original contexts, as differentiated from subsequent enculturation), and involves Exner's area in the activity that distinguishes a literate brain.

Literacy can certainly be acquired by individuals who lack typical sensorimotor abilities. Language has the potential for cross-modality in the sensory channels it exploits, not just audition but vision and touch. Acquiring literacy through touch or gesture (the latter can also mean without ever hearing the sounds of speech) attests to the plasticity of the brain in performing language functions, a quality that also informs literacy. Acquiring literacy without the mobility needed to produce handwriting is also possible. However, in both cases, original contexts without literacy must be differentiated from subsequent enculturation into existing literate systems. Literacy of the sort we experience could not have developed without audition and manuovisual abilities for making and refining marks in materials like clay. Handwriting is necessary to establishing associations between visible marks and language (were this not the case, literacy would more consistently develop where all such marks are made, regardless of the movements involved), as audition is critical to the phonetic component of scripts, vision to the recognition, refinement, and productive combination of characters.

In ontogenetic acquisition of established literacy, by comparison, immobility does not preclude participation, nor does brain functionality necessarily differ between mobile and immobile participants. In other words, when hand/arm movements are not possible, Exner's area likely performs motor-movement planning nonetheless, just as it does when the movements are also carried out. This is true in numeracy, where the brain region involved in finger representations and movements (angular gyrus) interacts with the region that appreciates quantity (intraparietal sulcus), even when the fingers do not move (Hatano et al. 1977; Roux et al. 2003), and it is implicit in rehabilitative therapies for stroke patients that involve imagined motor movements (Johnson 2000; Schwartz 1999).

\section{Materiality in literacy: Enabler of production, use, and change}


ANE individuals could certainly have learned to read but not to write; indeed, 'there are more attestations of reading than of writing on the part of nonprofessionals' (Charpin 2000, 66; emphasis in original). Such tangential participation, however, would have contributed only marginally to the manipulation of the writing system, with a correspondingly negligible influence on improving its expressiveness. Achieving fidelity required continual interaction between the materiality of writing and its various communities of users over time, allowing (proto-)cuneiform to change in ways that would ultimately yield an expressive script. This interaction affords an opportunity to mention the role of materiality in literacy. Materiality-writing surfaces and implements to mark them, as well as the forms of individual characters-allows writing to be produced and reading to occur; indeed, it is difficult to envision writing and reading apart from its materiality (Haas 1996). Several material forms, particularly clay and stone but also metal, wood, and possibly dust, were used to record and communicate information in the ANE; clay and stone have particularly dominated discussions about the origins of writing (Fig. 4):
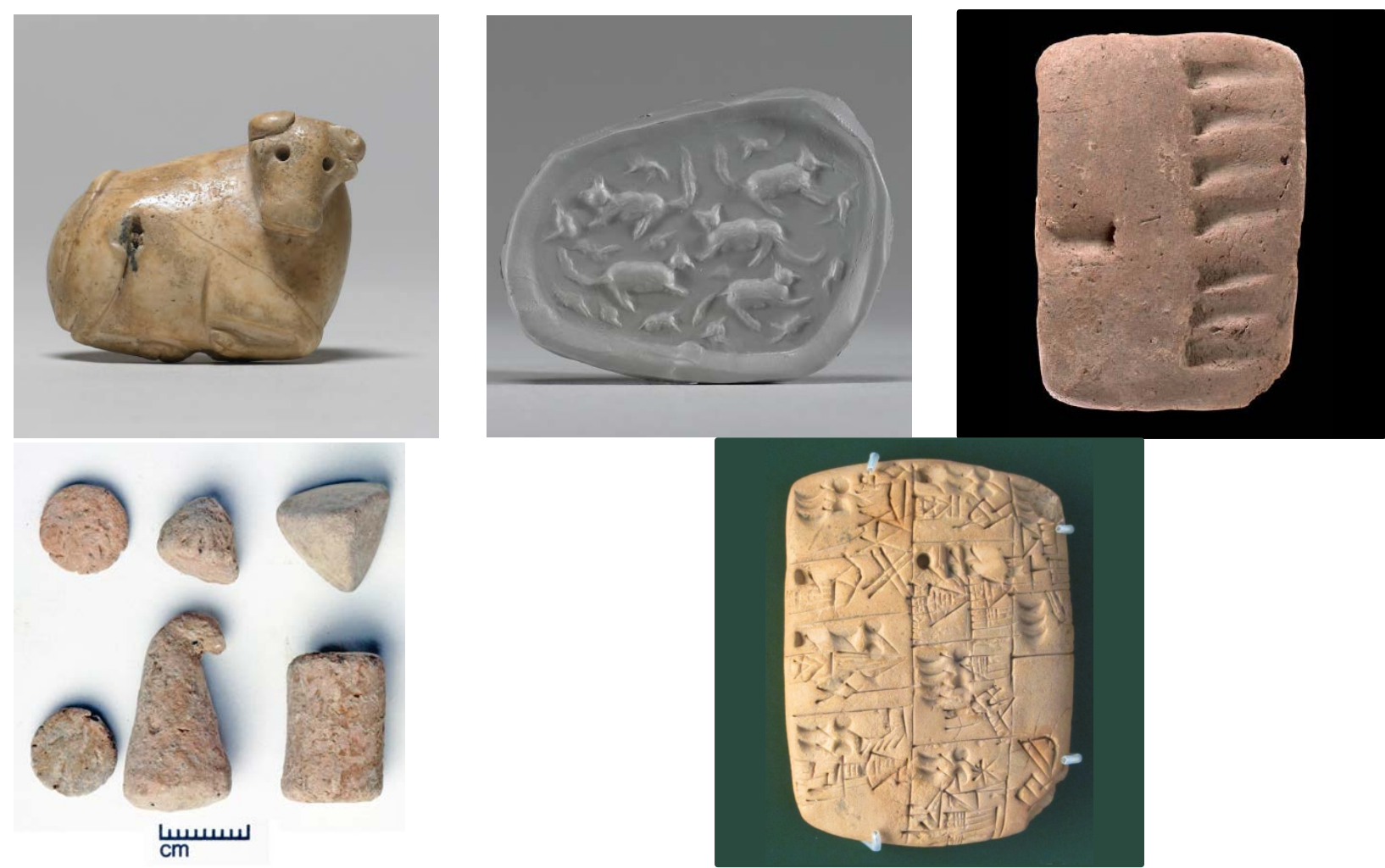

Figure 4. Top, left: Stamp seal in the form of a reclining bull (42.540) dated to 3200-2900 BC; acquired by Henry Walters (1928). Top, middle: The seal's impression depicts five large felines and other animals. Images courtesy of the Walters Art Museum, Baltimore (MD). Top, right: Clay tablet from Uruk (VAT 15261) dated to 3500-3350 BC with two columns of numerical impressions for quantities of grain. (C) Staatliche Museen zu Berlin Vorderasiatisches Museum; Foto: Olaf M. Teßmer. Bottom, left: Possible clay tokens dated to the first millennium (MacGinnis et al. 2014). Image courtesy of the Ziyaret Tepe Archaeological Expedition. Bottom, right: Clay tablet with numerical impressions and picto-/ideographs recording the allocation of beer (ME 140855) dated to 31003000 BC. Image courtesy of the British Museum under creative common licensing.

- Seals may have been the 'earliest representative and first precursor' of communicating 'abstract, intangible and conventional' ideas like ownership, possession, or authenticity 
through two-dimensional representations (Shendge 1983, 122). Stamp and cylinder seals were small, intaglio-engraved devices of hard material (often stone) used to imprint clay with images of animals, objects, or scenes, with stamp seals used possibly as early as the mid-sixth millennium. They sometimes also contained glyphs (or elemental signs).

- Numerical notations and their precursors, clay tokens, are thought to have led to the origins of writing (Schmandt-Besserat 1992; the idea that the so-called complex tokens precursed later written signs remains the most controversial aspect of this hypothesis). Numerical notations appeared in the archaeological record in the late-fourth millennium as impressions made by objects or fingers on clay bullae and envelopes (later, by styli on tablets), while possible tokens in the form of simple geometric shapes have been found throughout the region from the ninth to the first millennia (e.g. MacGinnis et al. 2014).

- Pictographs and ideographs, 'figurative and conventional drawings intended to have a communicative function,' also developed in the late-fourth millennium and perhaps comprised 'a system of signs that evolved slowly toward an approximate notation of the spoken language before it converted itself into a true phonetic system that integrated all linguistic dimensions' (Glassner 2000, 84, 215).

These technologies were closely connected: It is not unusual to find them in the same archaeological context. Seals, numerical notations, and/or picto-/ideographs often imprinted the same artefact, which may have also contained tokens. These technologies were additionally associated in their invention and representational capabilities: Some tokens are thought to have precursed the earliest numerical notations and writing by representing quantities (through repetition and bundling) and commodities (through shape and size); some complex tokens are thought to have precursed archaic picto-/ideographs (Amiet 1972; Schmandt-Besserat 1992; but see Englund 1998); and picto-/ideographs were used to label proto-cuneiform numerical notations (Krispijn 2012). While not minimizing the connections between these technologies, seals, tokens and numerical notations, and picto-/ideographs are considered here as distinct materials for writing, as they can be differentiated by properties like the hand movements needed to produce them.

An important differentiation must be made between general precursors and the specific circumstances producing literacy. All the technologies discussed in writing origins reflect societal motivations for recording and communicating information, behaviours of marking clay, and technological factors like the use of materials with suitable characteristics (e.g. manipulability, durability, availability). All these technologies transmitted cultural ideas through images (e.g. Wengrow 2014), designs, signs, and/or forms (both two- and three-dimensional). Thus, all precursed literacy, though not in any sense of linear evolution. Even more critical to developing literacy than the ability to communicate information is the ability to form associations between language and visually 'representable or reproducible form[s]' (Shendge 1983 , 113) that convey semantic and phonetic information. As argued here, only picto/ideographs had this outcome because only they satisfied all the circumstances needed to produce the necessary changes in both brains and writing.

As a writing surface, materiality both enables and constrains the shape a script ultimately takes. Clay, for example, not only requires particulates fine enough and adequately free of impurities to provide a cohesive, smooth writing surface ('levigation'), it must also contain enough moisture to remain malleable but not so much that it loses the firmness needed to hold impressions legibly (Taylor 2011). Clay dries quickly, providing relatively limited opportunities for 
modifications and corrections (Charpin 2000), possibly contributing to the remarkable conservation that typified the form, content, and organization of texts over several millennia. Straight lines are more easily made in clay than curved ones, contributing to the way that characters were made and picto-/ideographs developed into abstract forms. Complex marks in clay can quickly become an undifferentiated confusion of superimposed impressions (through "wedgeto-wedge interaction[s]"; Cammarosano 2014, 79), necessitating that characters be simplified and made with deliberation. In turn, simplicity improved speed of production, while deliberation improved legibility and reduced scribal errors. Simplicity also influences character production at a more basic level: characters taking hours to make do not record or communicate information efficiently because of their restricted volume (unless, like an image on a seal, they too could be stamped or rolled), nor would they facilitate the recombination needed for producing new signs.

Other materials beside clay, including stone and dust, were unlikely to have been initial media for literacy, either because they did not involve handwriting or were too ephemeral. A common material for seals and, by the late-second millennium, for documents for which 'there was a desire to confer a particularly solemn character' (Charpin 2000, 72), stone was shaped by lapidary techniques (Sax et al. 2000), movements distinct from handwriting. Further, the production of seals and even solemn documents would not have repeated characters in a way that produced handwriting effects. Dust, suggested as a medium for an abacus-like organization of numerical tokens (Høyrup 2000; Nissen et al. 1993), also did not involve handwriting, and moreover was highly perishable, unsuitable for recording or communicating information across time or distance. Clay, by comparison, was easily marked, adequately durable, and readily available. Such characteristics undoubtedly motivated the use of clay, which additionally necessitated that motor movements be fine and hand and eyes be coordinated to produce legible characters in it, generating handwriting effects and informing the use of wedges that epitomizes cuneiform.

If feature recognition entails that scripts can take a wide variety of forms, for writing to function as a script, it must be capable of representing semantic and phonetic elements of language. A writing system that can 'use visible marks to represent elements of a specific spoken language' (Sampson 1999, 895) is glottographic. In comparison, non-glottographic writing 'can be read with similar facility by speakers of different languages, or ... its reading has the character of paraphrase (i.e. two different 'readings' are likely to employ significant differences in word choice of syntactic construction)' (Hyman 2006, 234). Examples include Kanji as used by the foreigner, as well as the notations of mathematics and music (Gelb 1980; Powell 2009). ${ }^{7}$ The picto-/ideograms of archaic proto-cuneiform were similar in this regard: they were signs whose semantic meaning could be understood independently of language and which lack any clues to associated phonetic values. Kanji, for those trained to read it, contains both semantic and phonographic elements that identify, respectively, meaning and pronunciation (Hyman 2006). The latter is phonography, the notation of language sounds, which can be accomplished in several ways (Hyman 2006): by including (as Kanji does for literate individuals) an element that specifies how the sign is to be pronounced; by representing syllables with signs (e.g. katakana); and by employing the rebus principle ${ }^{8}$ to yield homophones. All these phonographic techniques are thought to have been used in archaic proto-cuneiform, though the evidence for them is slim and not uncontroversial (Hyman 2006). Further, the use of any such technique is related to the represented language (i.e. the utility of the rebus principle is related to the prevalence of homophones and near-homophones), and both language and culture more generally inform the selection and adaptation of images to represent words. 


\section{Production demands}

Earlier, writing and reading were represented as an interactivity of brains, bodies, and materiality, literacy as a change emerging from the interactivity nexus. The neuroscience of literacy, the involvement of bodies, and the demands of materiality suggest the following more detailed description of the interactivity: writing is repeatedly moving the hand to produce marks and visually judging them for legibility in a material that influences how movements are made and characters formed. Over time, this interaction improves hand-eye coordination, trains the fusiform gyrus to recognize written objects by their features, and increases coordination between the fusiform gyrus and the brain regions that comprehend and produce language and control handwriting movements. The changes in neurofunctionality inform changes in the materiality of writing, allowing it to become more expressive of language, which in turn intensifies neurofunctional change. With the contributions of brains, bodies, and materiality in mind, it is now possible to say something about the specific set of circumstances needed to produce literacy, where not even the concept of it had existed before (Table 1):

\begin{tabular}{lllll}
\hline & \multicolumn{3}{c}{ Technology } \\
\cline { 2 - 5 } \multicolumn{1}{c}{ Criteria } & Seals & Tokens & Numerical & notations \\
\hline Conventional & Some & Yes & Yes & Pictographs \\
Handwritten & No & No & Later & Yes \\
Repeated & Some & Yes & Yes & Yes \\
Simple & Some & Yes & Yes & Yes \\
Non-numerical & Yes & No & No & Yes \\
\hline
\end{tabular}

Table 1. Criteria for developing literacy. ${ }^{*}$ Numerical notations were initially produced by impressing objects or fingers into clay; they were later produced by making impressions with styli.

- Conventional. There must be an initial repertoire of signs with socially agreed meanings that record and communicate information. Such signs took various forms: images, designs, and glyphs on seals; numerical and complex tokens; numerical notations made with impressions and writing; and picto-/ideographs.

- Repeated. Sign-making must be repeated often enough that the motor activity trains object-recognition processes, engages handwriting effects, and allows writing to acquire greater expressiveness (hours per day; days over time). Many societies have independently developed writing, but only a few have developed a script and become literate; the latter appears to happen only when the repetition involved is that of a statelevel bureaucracy (e.g., Mesopotamia, Egypt, China, and Mesoamerica).

- Handwritten. Sign-making must involve precise motor movements of the hand in a way that reorganizes the brain and manipulates writing into script. This criterion eliminates seals and tokens, as they were not handwritten in either their production or use. Reading alone would be insufficient, since non-writing individuals would not participate in the manipulation of written forms needed to yield a script.

- Simple. The signs must be simple enough that they can be produced, repeated, and recombined. Production of characters is influenced by the materiality used. Simplicity enables the repetition needed to train object-recognition processing, engage handwriting 
effects, and develop a script. Recombination or productive combination allows marks to be adjusted towards greater meaningfulness and complexity, while minimizing ambiguity, qualities difficult to achieve with a relatively static system like the images of seals.

- Non-numerical. The signs cannot be numerical because numerals in and of themselves, no matter how much their production involves conventionality, repetition, handwriting, and simplicity, will not lead to literacy, a claim explained below. This criterion eliminates numerical notations.

\section{Why not numerical notations?}

Why would numerical notations not have led to literacy in Mesopotamia, despite their strong identification (along with tokens) with early writing by scholars of the ANE? Numerical notations certainly had all the right ingredients: they were conventional, repeated, eventually written by hand, and simple. They certainly recorded and communicated information in a way that precursed literacy. Making them would have trained the fusiform gyrus to recognize numerals by their features, enabling them to lose iconicity, as our own signs for ' 2 ' and ' 3 ' no longer resemble simple vertical strokes. Lexical words for numbers would undoubtedly have been associated with the notations (and the tokens that preceded them), consistent with the complexity of the represented number system. ${ }^{9}$ Numbers also overlapped with language, in both spoken and written form: they represented both words and syllables (Veldhuis 2011) and were used as nouns and adjectives (ordinal numbers) and grammatical markers (grammatical number) in third-millennium texts.

Nonetheless, despite the right ingredients and significant overlaps, there are multiple reasons to think that numerical notations, in and of themselves, do not cause literacy, and their contrast with written non-numerical language serves to illuminate properties important to its development:

- Literacy and numeracy, though often equated, are not the same: literacy is the ability to read and write, numeracy the ability to reason with numbers. Though they overlap in functionality and resources, their severability is attested by the fact that many societies develop numerical notations without developing scripts or literacy, a few scripts and literacy without numerical notations (Chrisomalis 2010).

- Relatedly, as material instantiations of numbers, numerals need not be written (like a tally records and communicates numbers and an abacus enables their manipulation). Literacy, in contrast, does not exist without it (Haas 1996), not just in the materiality involved but in handwriting as well.

- Numbers need only a few signs and thus represent a small subset of the total lexicon and phoneme inventory, likely insufficient to create literacy (Table 2 and Fig. 5).

- Language and numbers have very different universals (qualities shared across languages, cultures, and writing systems): Language is organized grammatically (Comrie 1989; Greenberg 1978), numbers by things like magnitude and intra- and inter-exponential structure (Chrisomalis 2010; Russell 1920) (Fig. 6). 


\begin{tabular}{llll}
\hline \multicolumn{1}{c}{ Writing } & \multicolumn{1}{c}{ Type } & \multicolumn{1}{c}{ Signs } & \multicolumn{1}{c}{ References } \\
\hline \multirow{3}{*}{ Scripts } & Logosyllabaries & Several hundreds & \\
& Syllabaries & $80-100$ & Daniels (1996) \\
& Alphabets & $20-35$ & \\
\hline Number systems & All types & $2-66$ & Chrisomalis (2010) \\
\hline \multirow{3}{*}{ Cuneiform } & Archaic (total, including numbers) & About 800 & \multirow{2}{*}{ Michalowski (1996) } \\
& Archaic (numbers only) & $60-70$ & Veldhuis (2011; 2014) \\
\hline
\end{tabular}

Table 2. Types of scripts and number systems with associated numbers of signs. Note. In writing systems, numbers of signs are distinctive enough to suggest script types. Logosyllabaries are writing in which 'the characters of a script denote individual words (or morphemes) as well as particular syllables'; in syllabaries, 'the characters denote particular syllables, and there is no systematic graphic similarity between the characters for phonetically similar syllables'; and in alphabets, 'the characters denote consonants and vowels' (Daniels 1996, 4). In the proto-cuneiform logosyllabary, most signs 'represented whole words ... [and] could function as syllables in other contexts (BA "ration" = /ba/)' (Michalowski 1996, 35). By comparison, numerical notation systems contain between two and 66 characters (Fig. 5). Archaic ANE writing falls within the typical range for logosyllabaries; its total numerical notations fall at the high end of the range for number systems, below the range for syllabaries, and above that of alphabets (which, notably, are a script type that develops from the other two). Thus, it is uncertain that any numerical notation system, including those of archaic proto-cuneiform, has enough characters (or the lexicon and phoneme inventories these represent) to develop literacy.

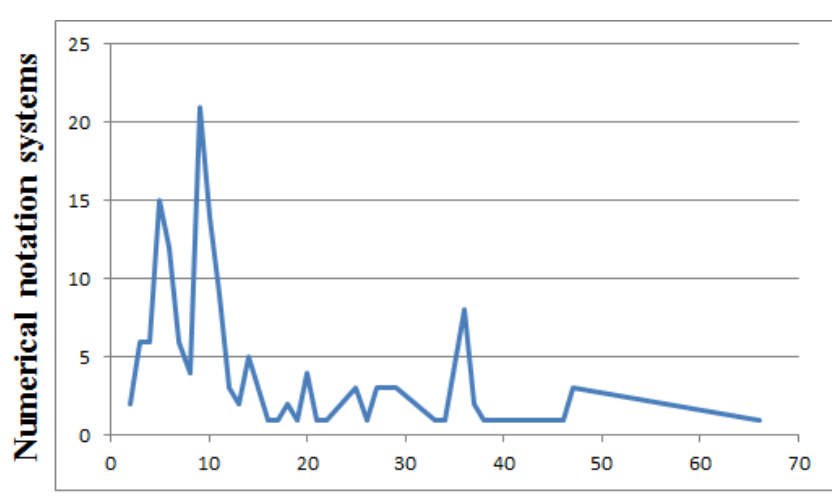

Characters per system
Figure 5. Character frequency in numerical notation systems, including archaic proto-cuneiform and later positional cuneiform numbers. The distribution is positively skewed by a small number of systems with larger-than-average sign sets; the average is 15 characters per system, the median about nine. Thirteen archaic proto-cuneiform number systems used a total of 62 characters with an average of five characters each (excluding fractions; with fractions, the total is 79, the average six). Sumerian cuneiform numbers were represented by five characters, Assyro-Babylonian four, and Babylonian positional only two (though the latter were accumulated in 14 patterns to express the numbers 1-59). Source data from Chrisomalis (2010).
Level of Morphology/Lexicon

\begin{tabular}{|cc|}
\hline CAT & 123 \\
*CTA & 132 \\
*ACT & 213 \\
*ATC & 231 \\
*TCA & 312 \\
*TAC & 321 \\
\hline
\end{tabular}

Level of Grammar and Syntax

\begin{tabular}{|ll|}
\hline John loves Mary & 123 \\
*John Mary loves & 132 \\
*Loves John Mary & 213 \\
*Loves Mary John & 231 \\
*Mary John loves & 312 \\
Mary loves John & 321 \\
\hline
\end{tabular}

Figure 6. Order serves different purposes in language and numbers, illustrated by how meaning changes when letters and numbers are identically reordered on the level of words (top) and sentences (bottom). Only some combinations on the left are well formed (those that are not are annotated with asterisks). In comparison, no matter how numbers are reordered, they remain meaningful, though the quantity they convey differs. In language, order forms words (morphology), phrases and sentences (grammar/syntax), and complex ideas and dialogue; in numbers, order denotes increasing magnitude, forms sequences, and gives '[n]ot only the integers, but also rational fractions and all real numbers ... most of their mathematical properties' (Russell 1920, 29). These and other differences between numbers and language entail that numbers can be rearranged all day long, but this activity will not suggest anything about features of language like word order-all that result are different quantities. 
- There are differences in how signs for numbers and words are processed visually and how they represent their meanings (Figs. 7 and 8).

- Numbers in material form are translinguistic as a function of how they are ordered and organized, while signs representing specific languages are ambiguous with respect to both semantic and phonetic values; such ambiguity entails that writing for language be elaborated, a mandate with no counterpart in numbers (discussed below).

5385383838384
misunderstood

\section{Visual Processing}

$$
\begin{gathered}
\text { 5,385,383,838,384 } \\
\text { m,isu,nde,rst,ood }
\end{gathered}
$$

Figure 7a. Numbers but not words benefit from visual separation. At left, the numbers are difficult to read, the word easy, because they are not separated; at right, the numbers are easier to read, the word more difficult, because they are separated. Recognition of words from local and global features is disrupted by separation; by comparison, numbers are read as linear sequences. In addition, some transposed letter and word arrangements might be correctly apprehended-or unnoticed-as a function of feature recognition (the phenomenon that makes it difficult to spot mistakes in proofreading; reading speed also decreases as errors increase: see Rayner et al. 2006). An analogy in cuneiform might be using a wrong sign with the same shape as the intended sign (a known type of scribal error). This has no parallel in numbers, where transpositions and substitutions yield the wrong quantity, not corrections that recover intended content.

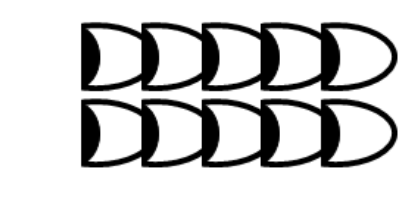

$$
\text { Ten } \mathrm{N}_{01}=\text { One } \mathrm{N}_{14} \quad \text { Eight } \mathrm{N}_{08}
$$

Figure 7b. In proto-cuneiform numerals, separation was a matter of bundling and grouping. Bundling reduced the amounts of each sign to a manageable number, while grouping arranged signs in a way that made their total quantity easier to discern. For example, ten $N_{01}$ (left, bottom) would have been bundled as a single $N_{14}$ (left, top), while eight $N_{08}$ (right) might have been grouped into two vertical lines of four.

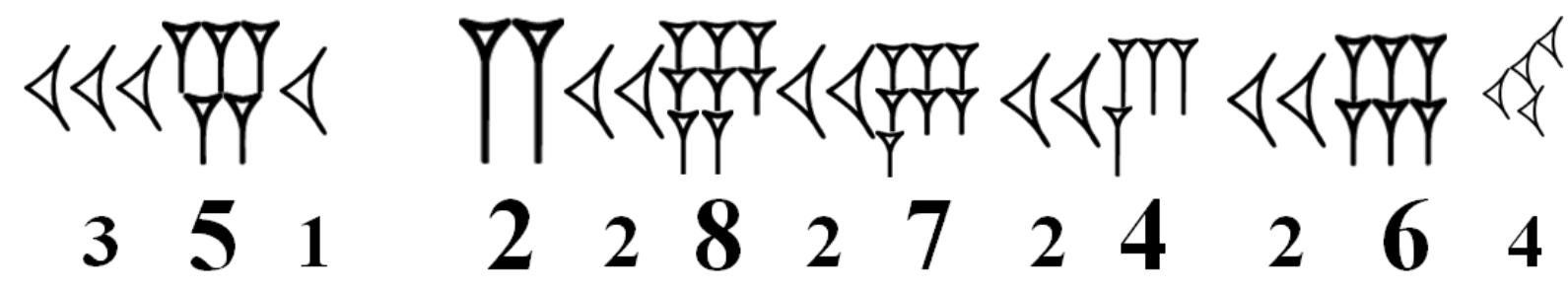

Figure 7c. Cuneiform numbers were bunched together (top). However, the use of alternating signs (tens and units) provided a kind of visual separation, similar in its effect to size differences (bottom), effectively separating larger units from smaller ones. The numbers are from the Plimpton 322 mathematical tablet (obverse, line 10: [1;]35;10;02;28;27;24;26;40), redrawn from Friberg (1981). 

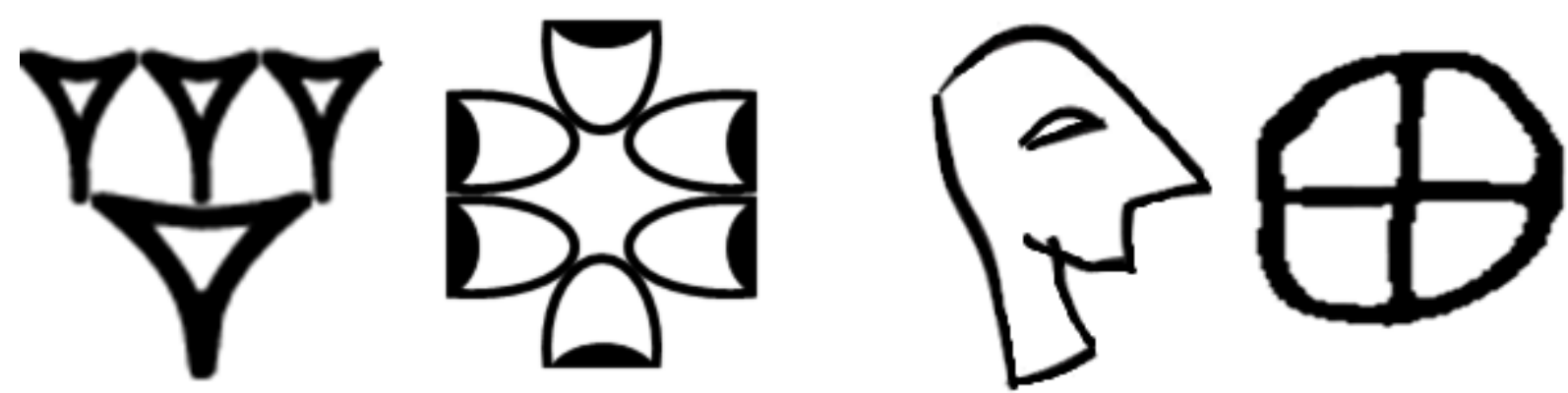

Figure 8. Cumulative notations (left) instantiate their referent (quantity), as four wedges are four in the integer 4, six bowls are six in the fraction 1/6. By comparison, pictographs (right, head) resemble their referents, ideographs (right, quartered disk) signify them, and both symbolize things related to them. This difference helps illustrate why language is ambiguous in what it communicates to a degree that numerical notations are not: notations for language are symbolic, allowing for mismatches between meanings and referents, while notations for numbers instantiate their meaning (i.e. with respect to quantity, they mean what they are). Signs adapted from the Cuneiform Digital Library Initiative.

Numerical notations are organized in ways common to all number systems (e.g. intraand inter-exponential organization; Chrisomalis 2010; also see Fig. 6). This gives numerical notations translinguistic recognizability and allows their representations to remain nonglottographic, as numerical signs' semantic value and shared organizational structure are sufficient (i.e. phonetic information is not essential). No such recognizability exists in other domains of language, where semantic meanings are relatively ambiguous and phonetic values unknown unless specified in some fashion. This difference entails that signs for non-numerical language must be elaborated through conventions that specify semantic and phonetic information to an increasingly precise degree: that is, notations for non-numerical language are under a pressure to become glottographic in a way that numerical notations are not. This same fundamental division appears in archaic proto-cuneiform: numerical notations were made by impressing clay with fingers, objects, or the blunt end of a stylus and were repeated to instantiate quantity, while non-numerical signs were incised with the sharp end of a stylus, not repeated, and depended on resemblance (pictographs), conventionality (ideographs), context of use (administrative), and organization (placement within cells on the tablet) for their intelligibility (Nissen et al. 1993; Powell 1971).

While both non-glottographic notations for numbers and glottographic notations for language submit their respective domains to 'reflection, analysis, and design' (Olson 1994, 258), they have very different purposes and outcomes: a sign for a number is a numeral, which records, communicates, and can-given the development of suitable algorithms - be used to manipulate quantity. In contrast, a sign for a word is a noun or verb or some other part of speech. The crucial distinction is this: numerals objectify numbers, not language. Whether in the form of numerical notations or tokens, no matter how often they are rearranged, numerals will not suggest things about features of language like word order and are thus unlikely to lead to a glottographic script, regardless of whether numbers are also used as nouns and adjectives or subsequently written with the same signs used for language (e.g. 'four', 'quatre', 'shi') once literacy has developed. Numerals remain non-glottographic because that form is sufficient for instantiating, representing, and objectifying numbers. Only picto-/ideographs, as nascent nouns and verbs, have a demand, and thus the potential, for being elaborated to represent the meanings and sounds of language in a less ambiguous manner. 
As notations for non-numerical language become elaborated to convey information about semantic and phonetic values, writing becomes a script that differentially engages the psychological processes involved in writing and reading, change that may further influence subsequent developments of writing expressiveness. Thus, the metaplastic interaction of the components of cognition allow change in both the system (which becomes literate) and its components (neurofunctional changes in the brain; increased expressive power in the script).

\section{Change in writing systems}

A discussion of how and why writing systems change is warranted because such change has the potential to index the development of literacy. Writing, as has been noted, originates as a system of markings with conventional references that communicate information (Powell 2009). Writing becomes a script, capable of expressing language with fidelity, through the interaction of psychological processes like object recognition with embodied behaviours like writing and materials like clay. Change could be inadvertent and incremental, arising from the interactivity of brains, bodies, and materiality as has been discussed, or directed and sudden, like the writing reform of the Ur III period (Michalowski 2006). How script change might index literacy is suggested by features like lexicography, dictionary-like compilations of words; organization, the layout of words upon the surfaces of writing materials; syntax, the ways in which characters, words, and phrases are arranged to reflect language; orthography, conventionalizations of signs and sign combinations; applications, the purposes to which writing and scripts are applied; curriculum, the systemization of training for the writing system; and language, the degree to which the writing system expresses an identifiable language. These are described below (Fig. 9):

- Lexicography. Vocabulary lists appear in the late-fourth millennium as word lists organized by theme (e.g. words for professions or traded goods). As mentioned, such lists were likely more codebooks than dictionaries. Sign order and form were highly conserved, attributes that would have facilitated their use as mnemonic devices (Wagensonner 2010) by archaic scribes with functional reading and writing but not true literacy. Lists organized by sign forms or other principles appear in the mid-third millennium (Englund 1998; Veldhuis 2014).

- Organization and syntax. Apart from numerical notations, which were ordered by increasing magnitude, little order was imposed on archaic signs used to label commodities, aside from their being placed in cases or cells (rectangular boxes) and put into columns on different tablet surfaces along with the numerical notations; effectively, they were labels identifying enumerated commodities in administrative tabulations (Charpin 2010; Englund 1994; Hyman 2006; Michalowski, 1996; Veldhuis 2012; 2014). Though by the mid-third millennium numerical notations would change form and in the late-third millennium develop positionality (Chrisomalis 2010), cuneiform numbers ordered quantity by increasing magnitude, the same way archaic ones did. For language, mandatory sign order began to appear in the mid-third millennium (Veldhuis 2012; 2014).

- Orthography. Proto-cuneiform was introduced in the late-fourth millennium, and chronologies of individual signs show them changing within a couple of centuries of use (Fig. 3). Changes in the first centuries of use suggest adjustments to improve character individuation and differentiation (respectively, visual abilities to identify any single character as itself and to tell one character apart from another: see Houston 2008), as well 
as relaxation of the need to preserve the original iconic form (Studevent-Hickman 2007). Syllabic signs appear in the mid-third millennium (Veldhuis 2012), about the same time that "words [were no longer" split between two lines (Cooper 1996, 45; however, date formulae continued to be an exception to this, perhaps as late as the Old Babylonian period). These changes likely represent handwriting effects and the recognition of written words by their features, allowing script characters to begin functioning as a system of contrastive elements, like morphemes and phonemes do in speech. ${ }^{10}$

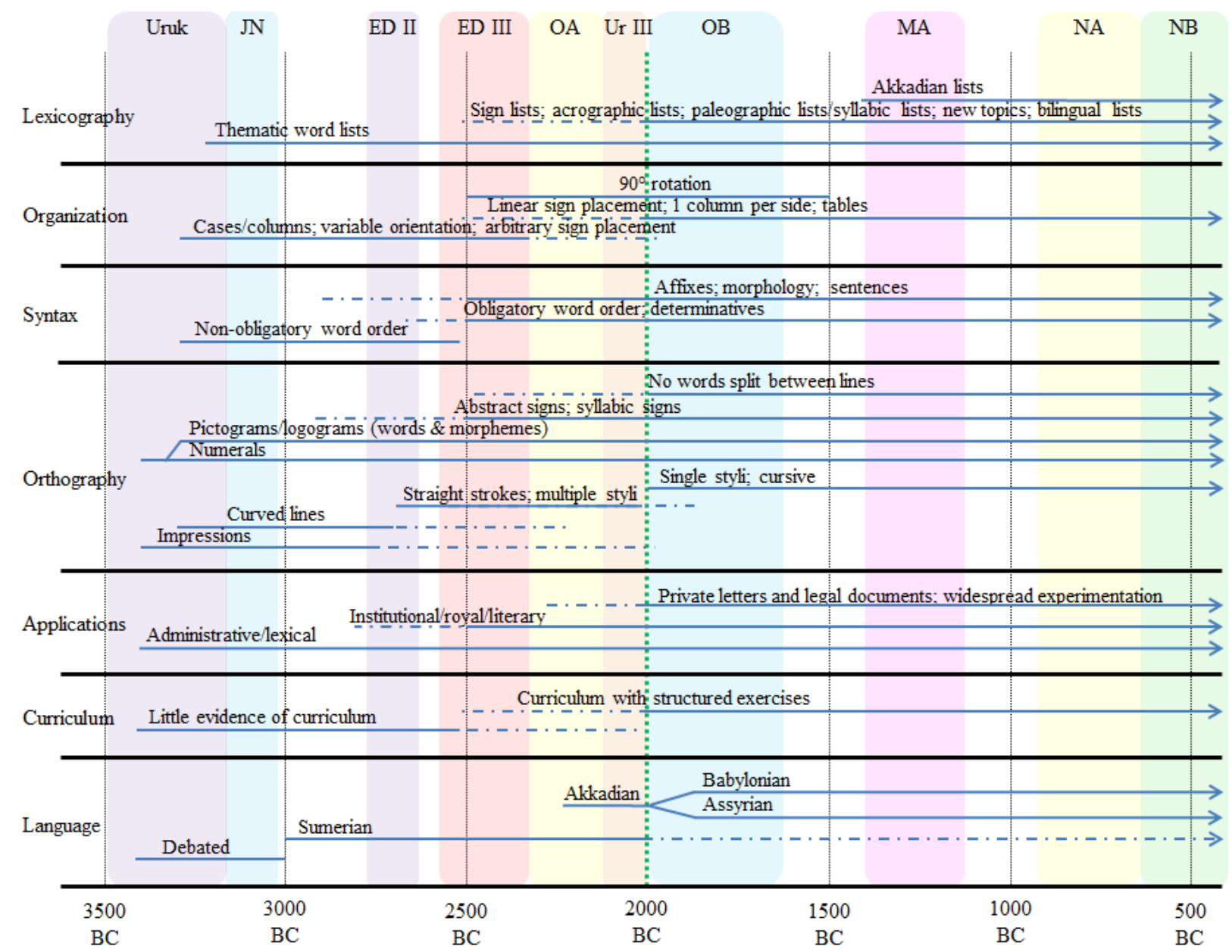

Figure 9. Summary of change in ANE writing in seven dimensions. The possible onset of true literacy is represented by the dashed vertical green line. Horizontal lines: Dashed = some evidence; solid = widespread evidence; the rightmost dashed line for Sumerian signifies that the language was no longer spoken but continued to be used in writing. $J N=$ Jemdet Nasr; $E D=$ Early Dynastic; $O A=$ Old Assyrian; OB = Old Babylonian; MA = Middle Assyrian; NA = Neo-Assyrian; NB = Neo-Babylonian. Sources of data: Charpin 2000; Cooper 1996, 2004; Englund 1998; Hyman 2006; Krispijn 2012; Schmandt-Besserat 1992; Taylor 2011; Veldhuis 2011, 2012, 2014.

- Applications. In the late-fourth millennium, writing was used strictly within administrative contexts. Institutional and royal writing characterized the third millennium (Veldhuis 2011), with literary texts appearing in the mid-third millennium (Veldhuis 2014). 
- Curriculum. From the late-fourth to the mid-third millennia, there is little evidence of an organized curriculum; structured exercises appear in the mid-third millennium (Veldhuis 2014).

- Language. Archaic proto-cuneiform had so little expressive power, scholars still debate its associated language. The Sumerian language was unambiguously expressed by the earlythird millennium, and writing was adapted for Akkadian toward the mid-third millennium.

Today, the ability to associate meanings and sounds of language with visual representations in an existing system of literacy is 'progressively acquired and becomes automatized typically after 3-4 years of training’ (Pegado et al. 2014, 1). Developing literacy in the absence of any pre-existing structures or an expressive script appears to take many centuries (Cooper 2004; Lorette 1999), as measured by comparing writing's first appearance to change in lexicography, organization and syntax, orthography, applications, and curriculum. Generally, the development of literacy may be indexed by:

- Lexicography. The incorporation of different organizational principles into word lists suggests an increased understanding of how writing reflects language.

- Organization and syntax. Significant decrease in the separation of word elements suggests a need for integrity that implies increased training of object-recognition processing; increased ordering of signs to reflect language suggests greater coordination between the fusiform gyrus and language centers of the brain.

- Orthography. Increasing abstractness of signs and development of cursive suggest handwriting effects and the training of object-recognition processing.

- Applications. The expansion of the purposes to which writing is applied suggests the increasing maturity of the literate system.

- Curriculum. Greater formalization of the training process would be necessitated by and implies the increasing opaqueness of script to non-initiates.

- Language. The achievement of identifiable language demonstrates significant increase in the script's expressive power.

These indices suggest change at the systemic level of the literate system, change implying that interactivity between components (brain, body, materiality) had influenced and intensified the transformation of writing into script. Broadly, manuovisual stimulation from the manipulation of material forms occasioned collective, cumulative change in psychological processing, which over the long term influenced brain functionality and form, and change in psychological processing facilitated the manipulation of materiality into new, stimulating forms with subsequent impact on neurological functioning - an interesting phenomenon, given that each new individual must acquire the skills needed to participate, and the long-term sustainment of behaviors required to develop the necessary psychological and material changes are socially motivated.

One obvious change is the $90^{\circ}$ rotation, regarding which three points must be highlighted. First, deciding when the rotation is correctly placed - the mid-third (Studevent-Hickman 2007), late-third (Powell 1981), mid-second (Picchioni 1980), and late-second millennium (Nissen et al. 1993) - is outside the scope of the present review. Second, scripts change for many reasons, influenced by (in no particular order) language, need for legibility, characteristics of materiality, ease of production, and random drift, as well as things like the size of the tablets, how they were held, and their positioning with respect to the stylus (Studevent-Hickman 2007), all possible and 
not mutually exclusive factors in the rotation. Third, by the time the rotation occurred, whenever and whyever it did, characters had become more abstract, something that may have 'made them more susceptible to the rotation' (Studevent-Hickman 2007, 489). This interpretation is reasonable, as increased abstraction suggests that signs were increasingly recognized by their features, and this may have facilitated subsequent changes to improve legibility and ease production.

It is not possible to determine whether all the changes across all the various dimensions represent causes or effects of interactions between the materiality of written forms and the neurofunctional changes in brains, or determine a precise chronology for the change trajectory. There is at present little neuroscientific theory or evidence on which to base a more detailed analysis of the interactions between psychological processes, embodied behaviours, and material forms in writing over the time it takes to create literacy. Developing these will require a revolution in neuroscience, one that incorporates materiality as an integral component of cognition, which in turn will necessitate significant adjustments to neuroscientific paradigms and research methodologies (e.g. Tallis 2011), as well as a way to reconcile vastly different time scales (psychology: milliseconds to decades; archaeology/texts: centuries to millennia). Script change is also often uneven (i.e. occurs faster in some regions), attributable to multiple causes (e.g. imposed by political upheaval or imperial decree; relocation of trained scribes), and cannot be assigned to any absolute chronology (as is true of the $90^{\circ}$ rotation; a separate issue is that some texts are dated archaeologically, others relatively sequenced through paleographic methods). There is also little insight into cuneiform developments between the late-fourth and early-third millennia (Charpin 2010; Veldhuis 2012), a time of critical change in archaic writing. Finally, the adaptation of cuneiform to Akkadian in the mid-third millennium would undoubtedly have had an effect on the development of literacy, perhaps accelerating awareness of sound and structure (as the adaptation of katakana to English words serves to illustrate; Fig. 1).

Literacy appears to stir in the first half of the third millennium, when writing started to reflect identifiable languages; was well underway by the second half, when many of the changes had become more widespread; and had likely been fully realized by the beginning of the second millennium, perhaps contributing to the "revolution in writing [that] took place around 2000 BCE” (Veldhuis 2012, 3). The Old Babylonian period saw both dramatic expansion of the use of writing and significant decrease in the amount of systemic reorganization (Fig. 9), though by no means should it (or any other writing phase) be considered a final state, as it also saw the introduction of a "cursive script, with abbreviated signs, crowded writing, and unclear sign boundaries” (Veldhuis 2011, 72), characteristics indicative of greater tolerance of ambiguity in recognizing signs, a handwriting effect. If the Old Babylonian period was indeed truly literate, then it is possible to compare its characteristic writing with that of earlier periods and speculate the differences represent at least some of the changes necessary to transform the writing associated with functional reading and writing into truly literate script. It is also reasonable to conjecture that material changes affected brain function and form, that these neurofunctional changes would have affected behavior and informed changes in materiality, and that this interactivity would have been mutually reinforcing and opened up opportunities for further changes in associating visual marks with the meanings and sounds of language. Future research, especially comparisons of ANE literacy with script chronologies and developments in other early writing traditions (as much as they can be determined, given the paucity of early writing materials, especially in China and Mesoamerica), may ultimately gain more traction on matters like critical changes, temporal sequencing, and functional interdependencies beyond the rough outline sketched here. 
The fundamental division seen in writing numbers and language is consistent with the independence of numeracy and language, a characterization based on double-dissociability: one person can lose the ability to speak but can still reason with numbers; another can lose the ability to reason with numbers but can still speak (Brannon 2005; Varley et al. 2005), which suggests that neither cognitive process requires the other to function. Independence obviously does not preclude language and numeracy from working together in powerfully synergistic ways: numbers qua numbers are expressible in speech, languages incorporate numbers as parts of speech, and there is a large overlap between literacy and numeracy in their functionality (e.g. both can be expressed aurally, tactually, and visually, though language is initially aural while numbers are initially manuovisual) and the neural, physical, and material resources recruited to support it.

Bundling and multivalency, however, may challenge the idea that literacy and numeracy are thoroughly independent. Bundling, the consolidation of higher quantities used in the protocuneiform numerals systems, represents multiple units of one shape and size as a single unit of a different shape or size. Such consolidation presumably decreases the number of numerical signs (or precursor tokens, also bundled: see Nissen et al. 1993) needed to represent quantity, facilitating recognition. Multivalency is the ability of any particular sign to hold different meanings based on its context of use: a numerical sign might represent six smaller units in one context but ten in another (Robson 2008). Together, bundling and multivalency entailed that numerical notations were related to each other and to their context as well, in addition to representing meanings of quantity and commodity type. Signs for non-numerical language were similarly related to each other and their context, and ultimately represented both semantic meanings and sound values. This suggests that either the ability to form and represent such relations is transferred between the numerate and literate domains, or both domains are informed by an underlying capability to form such relations, something that requires further research.

The first alternative seems at odds with the severability of numeracy and literacy. Certainly, it is possible that the ability to construe relations between signs in one domain merely increases the likelihood of doing so in another, even one in which the universal principles vastly differ, without co-influence being required. In this regard, the tokens preceding numerical notations would have contributed to the development of literacy, as they were similarly bundled and multivalent (Amiet 1972; Le Brun \& Vallat 1978; Le Brun 1978; Damerow \& Meinzer 1995). The second alternative, however, implies possible linkage between the two domains that might be essential to achieving numeracy, literacy, or both.

\section{Conclusion}

When literacy is viewed as a change in cognition resulting from the interactivity of brains, bodies, and materiality, it can be seen emerging from societal motivations for recording and communicating information over space and time through the use of conventionalized signs, embodied activity of marking materials like clay, and use of materials with qualities like manipulability, durability, and availability. However, the differences between functional writing/reading and true literacy should not be taken to mean the latter is better than the former, merely that both entail different cognitive-behavioural potentials and outcomes (e.g. Olson 1994; 2013). Nor should the account of the transition imply the process was straightforward or foreordained, or exclude the possibility that specific changes may have increased the likelihood of subsequent change. 
When Mesopotamians handwrote marks on clay with sufficient repetition over time, their brains reorganized. As their brains reorganized, the system elaborated further in the forms of script change, writing fidelity to language, scribal training and professionalism, and writing utility beyond accounting. Arguably, the Mesopotamians would not have developed literacy if they had continued to use only seals, tokens, and numerical notations. Nonetheless, these technologies were an integral part of the socio-material basis for picto-/ideographs, which were less likely to have developed without such precursors. The precursor technologies, no matter how nonlinear the trajectory between them was in actuality, served to make the phenomenon of literacy much more realizable by people of average capabilities participating in routine tasksthe scribes, merchants, traders, and farmers involved in writing picto-/ideographs for the purposes of recording and communicating information. It was the cumulative effort of countless small tinkerings by average people in mundane activity that led to the development of literacy, an example of material culture distributing cognitive effort over time and space (Hutchins 1995) and bringing vast innovation into the realm of ordinary achievability.

\section{Acknowledgements}

An early version of this paper was presented at the $4^{\text {th }}$ annual Oxford Postgraduate Conference in Assyriology. I would like to thank the audience members for their insightful feedback. I would also like to thank Jacob L. Dahl, Kathryn Kelley, Denise Schmandt-Besserat, and Jerrold S. Cooper for their comments on these ideas, as well as Stephen Chrisomalis and two anonymous referees for their constructive review.

\section{Notes}

1. The term Mesopotamians is 'problematic, since the former inhabitants of that region did not designate themselves as such and did not think of themselves that way' (Charpin 2010, 15); however, alternatives (e.g. Sumerians, Babylonians) are even more so. Mesopotamians at least avoids the Sumerian question and is generally inclusive of the various languages, cultures, and locations associated with the region's early writing traditions.

2. Children first learning to read lack both training and practice; monks in scriptoriums had enough of both to be able to reproduce text without necessarily being able to comprehend it, creating many of the errors that fuel textual analyses (Ehrman 2005).

3. It may be more accurate to characterize these as a single change, as during the development of literacy, brain and writing change together. This contrasts with the acquisition of literacy using a script that is already expressive.

4. Script form remains constrained by properties impacting the discernibility of signs: character size, contrast of written elements with the writing surface, etc.

5. As script characters become more abstract, they also look more alike to the non-literate reader. For example, as a corpus, proto-cuneiform characters are highly variable in appearance. In comparison, characters of the Old Babylonian script appear relatively uniform. The latter requires training and familiarity with the subtle clues that individuate and differentiate characters that the former does not.

6. Notably, across languages, the word 'writing' originally meant 'scratch' or 'paint' (Senner 1989). These verbs not only suggest what writing is as an activity (and thus the behavior associated with its origins), they also characterize the materiality involved (i.e. things that can be scratched or 
painted) and say something about what writing is not-drilling or chipping (stone), shaping (clay), or drawing lines (dust).

7. For example, ' $1+1=2$ ' can be read 'eins plus eins ist zwei', 'one added to one yields two', or 'deux est la somme de un plus un’; musical notations can be described with similar variability.

8. A common illustration of the rebus principle uses pictographs (an eyeball, tin can, waves, and a female sheep) to represent the words 'I can see you'.

9. This is not the traditional view of the lexical numbers associated with proto-cuneiform. However, just as a language (Sumerian or other) would have been associated with proto-cuneiform lexical signs (Hyman 2006), lexical numbers would have been associated with the complex archaic number systems. Certainly, in emerging number systems, such vocabulary develops in conjunction with subitizable numbers ('one, two, three': see Menninger 1992), while the archaic number systems counted to much higher quantities (Nissen et al. 1993).

10. As static, imprinted images and solid geometric shapes, seals and tokens would not have been subject to these change mechanisms in the same way that picto-/ideographs and numerical notations would have been.

\section{References}

Adelman, J.S., S.J. Marquis \& M.G. Sabatos-DeVito, 2010. Letters in words are read simultaneously, not in left-to-right sequence. Psychological Science 21(12), 1799-1801.

Amiet, P., 1972. Mémoires de la délégation archéologique en Iran, Tome XLIII, Mission de Susiane. Paris: Librairie Orientaliste Paul Geuthner.

Bolger, D.J., C.A. Perfetti \& W. Schneider, 2005. Cross-cultural effect on the brain revisited: universal structures plus writing system variation. Human Brain Mapping 25(1), 92-104.

Brannon, E.M., 2005. The independence of language and mathematical reasoning. Proceedings of the National Academy of Sciences of the USA 102(9), 3177-8.

Cammarosano, M., 2014. The cuneiform stylus. Mesopotamia XLIX, 53-90.

Cantlon, J.F. \& E.M. Brannon, 2007. Adding up the effects of cultural experience on the brain. Trends in Cognitive Sciences 11(1), 1-4.

Carreiras, M., J.A. Duñabeitia \& M. Perea, 2007. Reading words, numb3r5, and \$ymßol\$. Trends in Cognitive Sciences 11(11), 454-5.

Carreiras, M., M.L. Seghier, S. Baquero et al., 2009. An anatomical signature for literacy. Nature 461(7266), 983-6.

Changizi, M.A. \& S. Shimojo, 2005. Character complexity and redundancy in writing systems over human history. Proceedings of the Royal Society of London B: Biological Sciences 272(1560), 267-75.

Charpin, D., 2010. Reading and Writing in Babylon, trans. J.M. Todd. Cambridge (MA): Harvard University Press.

Chomsky, N., 2012. Poverty of stimulus: unfinished business. Studies in Chinese Linguistics 33(1), 3-16.

Chrisomalis, S., 2010. Numerical Notation: a Comparative History. Cambridge: Cambridge University Press.

Cohen, L. \& S. Dehaene, 2004. Specialization within the ventral stream: the case for the visual word form area. NeuroImage 22, 466-76.

Coltheart, M., 2014. The neuronal recycling hypothesis for reading and the question of reading universals. Mind \& Language 29(3), 255-69.

Comrie, B., 1989. Language Universals and Linguistic Typology. 2nd edition. Chicago (IL): University of Chicago Press. 
Cooper, J.S., 1996. Sumerian and Akkadian, in The World's Writing Systems, ed. P.T. Daniels \& W. Bright. New York (NY): Oxford University Press, 37-57.

Cooper, J.S., 2004. Babylonian beginnings: the origin of the cuneiform writing system in comparative perspective, in The First Writing: Script Invention as History and Process, ed. S.D. Houston. Cambridge: Cambridge University Press, 71-99.

Damerow, P. \& H.-P. Meinzer, 1995. Computertomografische Untersuchung ungeöffneter archaischer Tonkugeln aus Uruk, W 20987, 9, W 20987, 11 und W 20987, 12. Baghdader Mitteilungen 26, 7-33.

Daniels, P.T., 1996. The study of writing systems, in The World's Writing Systems, eds. P.T. Daniels \& W. Bright. New York (NY): Oxford University Press, 3-17.

Dehaene, S. \& L. Cohen, 2007. Cultural recycling of cortical maps. Neuron 56(2), 384-98.

Dehaene, S. \& L. Cohen, 2011. The unique role of the visual word form area in reading. Trends in Cognitive Sciences 15(6), 254-62.

Dehaene, S., F. Pegado, L.W. Braga et al., 2010. How learning to read changes the cortical networks for vision and language. Science 330(3), 1359-64.

Diakonoff, I.M., 1976. Ancient writing and ancient written language: pitfalls and peculiarities in the study of Sumerian. Assyriological Studies 20, 99-121.

Ehrman, B.D., 2005. Misquoting Jesus: the Story Behind Who Changed the Bible and Why. New York (NY): Harper.

Englund, R.K., 1994. Archaic administrative texts from Uruk: the early campaigns. Cuneiform Digital Library. http://www.cdli.ucla.edu/

Englund, R.K., 1998. Texts from the Late Uruk period, in Mesopotamien: Späturuk-Zeit und Frühdynastische Zeit. Freiburg, Switzerland: Universitätsverlag, 15-233.

Friberg, J., 1981. Methods and traditions of Babylonian mathematics: Plimpton 322, Pythagorean triples, and the Babylonian triangle parameter equations. Historia Mathematica 8, 277-318.

Frost, R., 2012. A universal approach to modeling visual word recognition and reading: not only possible, but also inevitable. Behavioral and Brain Sciences 35(5), 310-29.

Gelb, I.J., 1980. Principles of writing systems within the frame of visual communication, in Processing of Visible Language 2, eds. P.A. Kolers, M.E. Wrolstad \& H. Bouma. (NATO Conference Series, Series III: Human Factors.) New York (NY): Plenum Press, 7-24.

Glassner, J.-J., 2000. The Invention of Cuneiform: Writing in Sumer. Baltimore (MD): Johns Hopkins University Press.

Greenberg, J.H., 1978. Generalizations about numeral systems, in Universals of Human Language, ed. J.H. Greenberg. Stanford (CA): Stanford University Press, 249-95.

Haas, C., 1996. Writing Technology: Studies on the Materiality of Literacy. New York (NY): Routledge.

Hatano, G., Y. Miyaka \& M.G. Binks, 1977. Performance of expert abacus operators. Cognition 5(1), 57-71.

Heimann, K., M.A. Umilta \& V. Gallese, 2013. How the motor-cortex distinguishes among letters, unknown signs and scribbles: a high density EEG study. Neuropsychologia 51, 2833-40.

Houston, S.D. (ed.), 2008. The First Writing: Script Invention as History and Process. Cambridge: Cambridge University Press.

Høyrup, J., 2000. A note on Old Babylonian computational techniques. Filosofi og Videnskabsteori på Roskilde Universitetscenter 1-5.

Hutchins, E., 1995. Cognition in the Wild. Cambridge (MA): MIT Press.

Hyman, M.D., 2006. Of glyphs and glottography. Language \& Communication 26, 231-49.

James, K.H. \& L. Engelhardt, 2012. The effects of handwriting experience on functional brain development in pre-literate children. Trends in Neuroscience and Education 1(1), 32-42. 
Johnson, S.H., 2000. Imagining the impossible: intact motor representations in hemiplegics. NeuroReport 11(4), 729-32.

Konnikova, M., 2014. What's lost as handwriting fades. New York Times, D1. http://www.nytimes.com/2014/06/03/science/whats-lost-as-handwriting-fades.html

Krispijn, T.J.H., 2012. Writing Semitic with cuneiform script: the interaction of Sumerian and Akkadian orthography in the second half of the third millennium BC, in The Idea of Writing: Writing across Borders, eds. A. de Voogt \& J.F. Quack. Leiden, The Netherlands: Koninklijke Brill NV, 181-218.

Le Brun, A. \& F. Vallat, 1978. L’origine de l'écriture à Suse. Cahiers de la Délégation Archéologique Française en Iran 8, 11-59.

Le Brun, A., 1978. La glyptique du niveau 17B de l'acropole (campagne de 1972). Cahiers de la Délégation Archéologique Française en Iran 8, 61-79.

Longcamp, M., M.-T. Zerbato-Poudou \& J.-L. Velay, 2005. The influence of writing practice on letter recognition in preschool children: a comparison between handwriting and typing. Acta Psychologica 119(1), 67-79.

Lorette, G., 1999. Handwriting recognition or reading? What is the situation at the dawn of the 3rd millennium? International Journal on Document Analysis and Recognition 2(1), 2-12.

MacGinnis, J., M.W. Monroe, D. Wicke et al., 2014. Artefacts of cognition: the use of clay tokens in a Neo-Assyrian provincial administration. Cambridge Archaeological Journal 24(2), 289-306.

Malafouris, L., 2013. How Things Shape the Mind: a Theory of Material Engagement. Cambridge (MA): MIT Press.

McCandliss, B.D., L. Cohen \& S. Dehaene, 2003. The visual word form area: expertise for reading in the fusiform gyrus. Trends in Cognitive Sciences 7(7), 293-9.

Menninger, K., 1992. Number Words and Number Symbols: a Cultural History of Numbers, trans. P. Broneer. New York (NY): Dover.

Michalowski, P., 1996. Mesopotamian cuneiform, in The World's Writing Systems, eds. P.T. Daniels \& W. Bright. New York (NY): Oxford University Press, 33-37.

Michalowski, P., 2006. The lives of the Sumerian language, in Margins of Writing, Origins of Cultures, ed. S.L. Sanders. (The University of Chicago Oriental Institute Seminars 2.) Saline (MI): McNaughton \& Gunn, 159-84.

Mueller, P.A. \& D.M. Oppenheimer, 2014. The pen is mightier than the keyboard: advantages of longhand over laptop note taking. Psychological Science 25(6), 1159-68.

Nakamura, K., W.-J. Kuo, F. Pegado et al., 2012. Universal brain systems for recognizing word shapes and handwriting gestures during reading. Proceedings of the National Academy of Sciences of the USA 109(50), 20762-7.

Nissen, H.J., P. Damerow \& R.K. Englund, 1993. Archaic Bookkeeping: Early Writing and Techniques of Economic Administration in the Ancient Near East. Chicago (IL): University of Chicago Press.

Olson, D.R., 1994. The World on Paper: the Conceptual and Cognitive Implications of Writing and Reading. Cambridge: Cambridge University Press.

Olson, D.R., 2013. Literacy and the languages of rationality. Pragmatics \& Cognition 21(3), 431-47.

Pegado, F., K. Nakamura \& T. Hannagan, 2014. How does literacy break mirror invariance in the visual system? Frontiers in Psychology 5, 1-5.

Pelli, D.G., B. Farell \& D.C. Moore, 2003. The remarkable inefficiency of word recognition. Nature 423(12), 752-6.

Perfetti, C.A. \& L.-H. Tan, 2013. Write to read: the brain's universal reading and writing network. Trends in Cognitive Sciences 17(2), 56-7.

Perfetti, C.A., 2003. The universal grammar of reading. Scientific Studies of Reading 7(1), 3-24. 
Picchioni, S.A., 1980. La direzione della scrittura cuneiforme e gli archivi di Tell Mardikh Ebla. Orientalia 225-51.

Powell, B.B., 2009. Writing: Theory and History of the Technology of Civilization. Chichester (UK): Wiley-Blackwell.

Powell, M.A., 1971. Sumerian Numeration and Metrology. Doctoral dissertation, University of Minnesota.

Powell, M.A., 1981. Three problems in the history of cuneiform writing: origins, direction of script, literacy. Visible Language 15(4), 419-40.

Price, C.J. \& J.T. Devlin, 2003. The myth of the visual word form area. NeuroImage 19, 473-81.

Rayner, K., S.J. White, R.L. Johnson et al., 2006. Raeding wrods with jubmled lettres: there is a cost. Psychological Science 17(3), 192-3.

Robson, E., 2008. Mathematics in Ancient Iraq: a Social History. Princeton (NJ): Princeton University Press.

Roux, F.-E., S. Boetto, O. Sacko et al., 2003. Writing, calculating, and finger recognition in the region of the angular gyrus: a cortical stimulation study of Gerstmann syndrome. Journal of Neurosurgery 99(4), 716-27.

Russell, B., 1920. Introduction to Mathematical Philosophy. 2nd edition. London: George Allen \& Unwin, Ltd.

Saggs, H.W.F., 1960. An unaddressed Old Babylonian letter. Journal of Cuneiform Studies 14(2), 56-58.

Sampson, G., 1999. Writing systems, in The MIT Encyclopedia of the Cognitive Sciences, eds. R.A. Wilson \& F.C. Keil. Cambridge (MA): MIT Press, 894-6.

Sax, M., N.D. Meeks \& D. Collon, 2000. The early development of the lapidary engraving wheel in Mesopotamia. Iraq 62, 157-76.

Schmandt-Besserat, D., 1992. Before Writing: From Counting to Cuneiform, 2 vols. Austin (TX): University of Texas Press.

Schwartz, D.L., 1999. Physical imagery: kinematic versus dynamic models. Cognitive Psychology 38, 433-64.

Senner, W.M., 1989. Theories and myths on the origins of writing: a historical overview, in The Origins of Writing, ed. W.M. Senner. Lincoln (NE): University of Nebraska Press, 1-26.

Shendge, M.J., 1983. The use of seals and the invention of writing. Journal of the Economic and Social History of the Orient 26(2), 113-36.

Studevent-Hickman, B., 2007. The ninety-degree rotation of the cuneiform script, in Ancient Near Eastern Art in Context: Studies in Honor of Irene J. Winter by Her Students, eds. J. Cheng \& M.H. Feldman. Leiden, The Netherlands: Brill, 486-513.

Sülzenbrück, S., M. Hegele, G. Rinkenauer et al., 2011. The death of handwriting: secondary effects of frequent computer use on basic motor skills. Journal of Motor Behavior 43(3), 247-51.

Tallis, R., 2011. Aping Mankind: Neuromania, Darwinitis and the Misrepresentation of Humanity. Durham (UK): Acumen.

Tan, L.H., A.R. Laird, K. Li et al., 2005. Neuroanatomical correlates of phonological processing of Chinese characters and alphabetic words: a meta-analysis. Human Brain Mapping 25(1), 83-91.

Taylor, J., 2011. Tablets as artefacts, scribes as artisans, in The Oxford Handbook of Cuneiform Culture, eds. K. Radner \& E. Robson. Cambridge: Cambridge University Press, 5-31.

Vanstiphout, H.L.J., 1995. Memory and literacy in ancient western Asia, in Civilizations of the Ancient Near East, ed. J.M. Sasson. Peabody (MA): Hendrickson, 2181-96.

Varley, R.A., N.J.C. Klessinger, C.A.J. Romanowski et al., 2005. Agrammatic but numerate. Proceedings of the National Academy of Sciences of the USA 102(9), 3519-24. 
Veldhuis, N., 2011. Levels of literacy, in The Oxford Handbook of Cuneiform Culture, eds. K. Radner \& E. Robson. Oxford: Oxford University Press, 68-89.

Veldhuis, N., 2012. Cuneiform: changes and developments, in The Shape of Script: How and Why Writing Systems Change, ed. S.D. Houston. Santa Fe (NM): School for Advanced Research, 3-24.

Veldhuis, N., 2014. History of the Cuneiform Lexical Tradition. Münster: Ugarit-Verlag.

Vogel, A.C., S.E. Petersen \& B.L. Schlaggar, 2014. The VWFA: it's not just for words anymore. Frontiers in Human Neuroscience 8, 1-10.

Wagensonner, K., 2010. Early lexical lists revisited: structures and classification as a mnemonic device, in Language in the Ancient Near East: Proceedings of the 53e Rencontre Assyriologique, eds. L. Kogan, N. Koslova, S. Loesov et al. (Annual of Ancient Near Eastern, Old Testament, and Semitic Studies.) Winona Lake (IN): Eisenbrauns, 285-310.

Wengrow, D., 2014. The Origins of Monsters: Image and Cognition in the First Age of Mechanical Reproduction. Princeton (NJ): Princeton University Press. 